\section{Importance of Contact Surface Between Electrodes and Treated Tissue in Electrochemotherapy}

\author{
WWW.tcrt.org
}

\begin{abstract}
Electrochemotherapy is an effective antitumor treatment employing locally applied high voltage electric pulses delivered through conductive electrodes to the tumor in combination with chemotherapeutic drugs. The efficiency of electrochemotherapy strongly depends on the local electric field distribution inside the target tissue. For successful therapy the entire target tissue has to be exposed to the local electric field strength above the reversible threshold. The aim of this study is to demonstrate the influence of the contact surface between electrode and treated tissue on the coverage of the tumor tissue by sufficiently high local electric field. The electric field distribution is calculated by means of numerical modeling using finite element method. Numerical results are confirmed with in vivo experiments. We demonstrated that the placement of electrodes giving larger electrode-tissue contact surface leads to improved electrochemotherapy outcome. Our results provide guidance on electrochemotherapy for treatment of protruding cutaneous tumors using parallel plate electrodes.
\end{abstract}

Key words: Electroporation; Electropermeabilization; Electrochemotherapy; Cutaneous tumor; Numerical modeling; Electrodes; DNA electrotransfer; Gene electrotransfer; Electric field distribution.

\section{Introduction}

Electrochemotherapy (1) (ECT) is a non-thermal antitumor treatment employing locally applied high voltage electric pulses (EP) in combination with either sistemic or local injection of chemotherapeutic drugs, such as bleomycin and cisplatin. ECT has been proven to be highly efficient in treatment of solid tumors regardless of their histological origin (1-7). The ECT Standard Operating Procedures are presently defined for cutaneous and subcutaneous tumors (8).

ECT is based on the reversible increase of cell membrane permeability that follows cell exposure to appropriate EP, termed electropermeabilization or electroporation (EPN). These EP, alone, do not kill the cells and have no antitumor effect. Indeed they just allow cytotoxic drugs, which otherwise do not easily penetrate cell membrane to enter and the target tumor cells and to kill them $(9,10)$.

An important advantage of ECT is that it results in the complete response (CR) of the tumors with drug doses that by themselves have minimal or no antitumor effect and no toxicity on the patients. After ECT good cosmetic effects are obtained due to a selective cell death mechanism that primarily affects the dividing tumor cells $(9,11)$.

Abbreviations: ECT, Electrochemotherapy; EP, Electroporation pulses; EPN, Electropermeabilization; CR, Complete response.
Selma Čorović, M.Sc. ${ }^{1,2,3, \$}$

Bassim Al Sakere, MD, M.Sc. ${ }^{1,2,8}$

Vincent Haddad, M.Sc. ${ }^{4}$

Damijan Miklavčič, D.Sc. ${ }^{3}$

Lluis M. Mir, D.Sc. ${ }^{1,2, *}$

${ }^{1}$ CNRS UMR 8121

Institut Gustave-Roussy

Villejuif, France

${ }^{2}$ University Paris-Sud

UMR 8121

${ }^{3}$ University of Ljubljana

Faculty of Electrical Engineering

Ljubljana, Slovenia

${ }^{4}$ Service of Biostatistics and Epidemiology

Institute Gustave-Roussy

Villejuif, France

${ }^{\S}$ Both authors contributed equally.

"Corresponding Author:

Lluis M. Mir, D.Sc.

Email : luismir@igr.fr 
In order to obtain a good antitumor effect it is mandatory to electropermeabilise the whole tumor. When a tissue is exposed to EP, an electric field strength is established within the tissue volume. To electropermeabilize the whole tumor in a reversible and safe way, each of the tumor cells have to be subjected to a local electric field $(E)$ above reversible value $E_{\text {rev }}$ (which causes transient and reversible perturbations in the cell membrane) and below irreversible value $E_{\text {irrev }}$ (which causes permanent damages of the cells). $E$ distribution depends on EP parameters (i.e., amplitude of the pulses, duration, number, repetition frequency, and shape), number, shape, and position of electrodes with respect to the treated tissue, structure of target/tumor tissue, and its surrounding tissues (12-15). It was also previously shown, by combining numerical modeling and experimental approaches, that the ECT treatment efficacy, at a given electrode configuration and pulse amplitude, depends on the magnitude of $E$ within the whole of the target/tumor tissue $(10,12-14,16-18)$.

In our present study, we numerically and experimentally analyzed the effect of two different ways of parallel plate electrode placement on the electric field distribution and on the efficacy of ECT treatment of cutaneous tumor with parallel plate electrodes. In the first situation, the electrodes were placed in such a way that very small contact with tissue was formed, while in
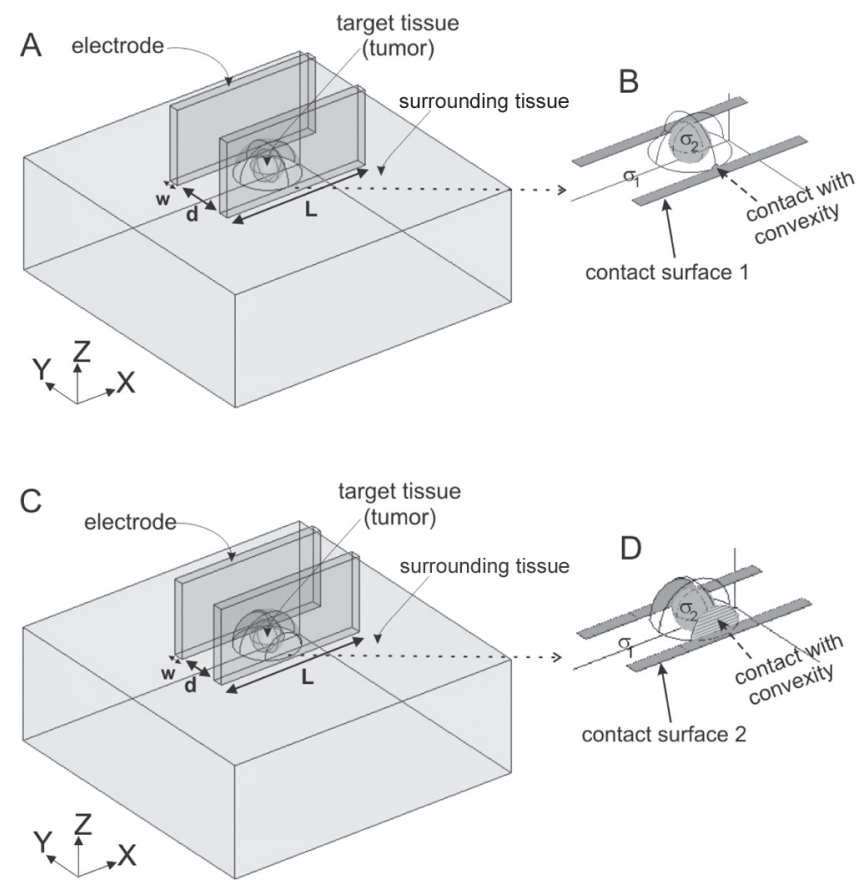

Figure 1: Panels $\mathbf{A}$ and $\mathbf{B}$ : Model of cutaneous tumor with $d=4 \mathrm{~mm}$, $U=520 \mathrm{~V}$ and contact surface 1 ; (A) $3 D$ model with electrodes and (B) zoomed convexity of the treated tissue with highlighted contact surface 1 . Panels C and B: Model of cutaneous tumor with $d=3 \mathrm{~mm}, U=390 \mathrm{~V}$ and contact surface 2: (C) $3 D$ model with electrodes and (D) zoomed convexity of the treated tissue with highlighted contact surface 2 . In both models $w=0.7 \mathrm{~mm}, \mathrm{~L}=10 \mathrm{~mm}, \sigma_{l}$ is surrounding tissue conductivity and $\sigma_{2}$ is tumor tissue conductivity. the second one the tissue was placed between two electrodes so that a large surface of contact between electrode and tissue was formed. Predictions on ECT efficacy were made according to the numerical results and they were confronted to the results of the in vivo ECT of mice tumors obtained by the two different electrode placements. We demonstrate that the placement of electrodes giving larger electrode-tissue contact surface results in better effectiveness of ECT outcome and in the lower effects on the surrounding normal tissue, a result important for the physicians applying ECT to their patients.

\section{Materials \& Methods}

\section{Numerical Modeling}

The 3D model is based on the numerical solution of partial differential equations for steady electric current in isotropic conductive media. The numerical calculations were performed by means of finite element method using COMSOL Multiphysics 3.3 software package in the 3D Conductive Media DC application mode on a PC running Windows XP with a $3.00 \mathrm{GHz}$ Pentium D processor and $1 \mathrm{~GB}$ of RAM.

The model of cutaneous tumor consisted of two tissues: the tumor and its surrounding skin tissue. The input to the model was constant voltage applied to the electrodes and corresponds to the amplitude of the EPN pulses generated by a high voltage generator. The output of the model was the electric field distribution within the treated tissues.

Two distinct model geometries representing two different parallel plate electrode placement situations were built (Fig. 1a and Fig. 1c). The convexity of the tumor and skin is modeled as a $4 \mathrm{~mm}$-diameter hemisphere and the tumor as a 2 mm-diameter sphere.

The results of numerical simulations were controlled by refining the mesh until the difference in numerical solutions was negligible (less than $0.5 \%$ ) when the number of finite elements (i.e., mesh density) is increased. The mesh of the models with contact surface 1 and contact surface 2 [models 1 (Fig. 1a) and 2 (Fig. 1c)] consisted of 176,419 and 158,760 elements, respectively.

The calculated electric field distribution in our numerical models is shown in the range between the critical values $E_{r e v}$ $=400 \mathrm{~V} / \mathrm{cm}$ and $E_{\text {irrev }}=1500 \mathrm{~V} / \mathrm{cm}$. The $E_{\text {rev }}$ is chosen based on our previous study (16), while the $E_{\text {irrev }}$ is chosen based on the latest study on irreversible EPN (18).

\section{Contact Surface Definition}

Model 1 (Fig. 1a) describes the treatment situation where electrodes are placed perpendicularly with respect to the sur- 
face of the cutaneous tumor touching the skin tissue just at the limit of the tumor convexity. Model 2 (Fig. 1c) describes the situation where the same cutaneous tumor is placed in between two electrodes touching also the surface of the volume convexity (see patterned region of the contact surface 2, Fig. 1d). In model 1 contact is obtained on a small surface of the treated volume convexity, pointed to by the dashed arrow in Figure 1b, corresponding to $2 \%$ of the contact surface in model 2. The distances between electrodes $(d)$ were $4 \mathrm{~mm}$ and $3 \mathrm{~mm}$, respectively. The voltage delivered to the tissue from the EP generator was modeled as a constant potential set to the entire surface between electrodes and treated tissue. In both models the applied voltage was set so that the ratio $U / d=1300 \mathrm{~V} / \mathrm{cm}$ was obtained, setting the voltage to $520 \mathrm{~V}$ and $390 \mathrm{~V}$, respectively, in model 1 and 2. Based on this the Direchlet boundary condition was defined. The Neumann boundary condition, i.e., the electric-insulation condition was set to the rest of the outer boundaries of the models.

\section{Material Properties of the Modeled Tissues}

Since we modeled the tumor and surrounding skin layers at the end of the EPN process (to numerically evaluate the outcome of the treatment), the conductivity values of tumor and its surrounding tissue were selected considering both data obtained with direct current measurements of tissue conductivities of electropermeabilized tissues (19) and the conductivity data obtained in in vivo experiments and numerical analysis in (16). Namely, conductivities of $0.4 \mathrm{~S} / \mathrm{m}$ and $0.2 \mathrm{~S} / \mathrm{m}$ were assigned, respectively, to the regions representing the electropermeabilized tumor and the electropermeabilized surrounding tissue (which describes the average conductivity of the skin tissue, not considering the high resistance of the stratum corneum that is almost immediately broken after the beginning of the first EP) (20). Thus, both tissues in our model were considered to be isotropic and homogeneous, not taking into account the changes in conductivity of tissues due to electropermeabilization. These changes would require an additional more complex analysis of functional dependency of tissue conductivity on electric field distribution, while not contributing to the demonstration of the influence of the contact surface on the ECT outcome.

\section{In Vivo Experiments}

The LPB cells, a methylcholanthrene-induced C57B1/6 mouse sarcoma cell line, was cultured using standard procedures and minimum essential medium (Gibco BRL, CergyPontoise, France) supplemented with 100U/ml penicillin, $100 \mathrm{mg} / \mathrm{ml}$ streptomycin (Sarbach, France), and $8 \%$ foetal calf serum (Gibco). C57Bl/6 female mice, 6-8 weeks old, were inoculated subcutaneously in the left flank with $1 \times 10^{6}$ cells, producing cutaneous tumors of the same dimensions as those of the numerical models in about nine days.
The two longest orthogonal diameters of the cutaneous tumor $a$ and $b$ (with $a>\mathrm{b}$ ), were measured using callipers. At the day of treatment, average tumor diameter, including the skin tissue was $3.85 \pm 0.21 \mathrm{~mm}$. The range of the tumor diameters $(a+b) / 2$ was $3.5-4.25 \mathrm{~mm}$ and the maximal individual diameter $a$ was $4.3 \mathrm{~mm}$. The volume $V$ of the tumors was determined using the Equation [1]:

$$
V=(\pi / 6) \cdot a \cdot b^{2}
$$

Animals were housed and handled according to recommended guidelines (21).

\section{Tumor Treatment and Follow-up}

Mice were anaesthetised with $12.5 \mathrm{mg} / \mathrm{kg}$ xylazine (Bayer Pharma, Puteaux, France) and $125 \mathrm{mg} / \mathrm{kg}$ ketamine (Parke Davis, Courbevoie, France) injected intraperitoneally in 150 $\mu \mathrm{l}$ saline before the ECT.

ECT treatment consisted in an intravenous injection of 10 $\mu \mathrm{g}$ bleomycin (Roger Bellon, Neuilly, France) followed, 4 minutes after the injection, by 8 rectangular, $100 \mu$ s long $\mathrm{EP}$ at a repetition frequency of $5000 \mathrm{~Hz}(2,22)$. The EP were generated by a Cliniporator ${ }^{\mathrm{TM}}$ (Igea, Carpi, Italy) and delivered directly to the tissue using $3 \mathrm{~mm}$ and $4 \mathrm{~mm}$ apart stainless-steel parallel plate electrodes $(10 \mathrm{~mm}$ long and 0.7 $\mathrm{mm}$ wide). The electrodes were placed in direct contact to the previously shaved skin on both sides of the cutaneous tumors. No conductive gel was used, to fully respect the geometry and contact area between the skin and the electrodes. As in the models described before, the amplitude of voltage pulses applied between the two parallel electrodes 3 and $4 \mathrm{~mm}$ apart were $390 \mathrm{~V}$ and $520 \mathrm{~V}$, respectively. The ECT outcomes obtained with these two electrode placements were followed in two groups of 15 mice for 27 days after the ECT, by means of measurements of the perpendicular diameters $a$ and $b$ every second day. A control group of 13 mice, not treated with ECT, was similarly followed. Response to ECT was qualified as $\mathrm{CR}$ if tumor completely disappeared at the end of the follow-up. For ethical reasons all the mice bearing tumor were sacrificed at the end of the follow-up, while those having no palpable or visible tumor were observed up to day 100. No tumor recurrence was observed at the end of the observation period. In addition very good cosmetic healing scar was obtained in the mice which responded with CR.

\section{Statistical Analysis}

The normal distribution of the individual tumor volumes was verified by the test of Shapiro-Wilk and a linear model was then used to determine the significance of the differences between the evolutions of the tumor volumes in the various experimental groups. The Fisher's exact test was 
used to compare differences in CR rates between each individual group treated with ECT and the control group of mice and between the two groups treated by ECT.

\section{Results}

\section{Numerical Results}

The comparison of the numerically calculated $E$ distribution between the two $3 D$ models of cutaneous tumor in $X Y$ $(Z=0), Z Y(X=0)$, and $Z X(Y=0)$ planes is shown in Figure 2. The contact surfaces 1 and 2 are given in Figures $2 \mathrm{a}$ and $\mathrm{b}$, respectively. The $X Y, Z Y$, and $Z X$ planes are three orthogonal cross-sections along the center of the tumor geometry. The electric field distribution within model 1 is shown in Figure 2a1-3 and within model 2 in Figure 2b1-3. The values of $E$ are displayed in the range from $E_{\text {rev }}=400$ $\mathrm{V} / \mathrm{cm}$ to $E_{\text {irrev }}=1500 \mathrm{~V} / \mathrm{cm}$ value (scale bar, Fig. 2 ). The patterned region represents the part of tissue with electric field strength exceeding the $E_{\text {irrev }}$.

The comparison of $E$ presented in Figures 2a1 and 2a 2 and in Figures $2 \mathrm{~b} 1$ and $2 \mathrm{~b} 2$ shows that $E$ within the tumor inside model 2 is higher and more restrained within the tissue area situated between electrodes.

Comparing the $X Y$ cross-sections, $E$ exceeds $E_{\text {rev }}(400 \mathrm{~V} /$ $\mathrm{cm}$ ) in a very small part of the tumor in model 1 (Fig. 2a1), while the entire tumor in the model 2 is subjected to $E>$ $400 \mathrm{~V} / \mathrm{cm}$ (Fig. 2b1). The highest $E$ within the tumor surrounding tissue is in the close proximity to the electrode, decreasing toward the center of the tumor in $Y$ direction. In the $X$ direction $E$ decreases symmetrically towards the two marginal tissue regions.

Comparing the $Z Y$ cross-sections, $E$ inside model 1 (Fig. 2a2) only exceeds the $E_{r e v}$ value in the deepest part of the tumor, while the upper part remains below $E_{\text {rev }}$. In model 2 the entire tumor tissue is covered by $E>E_{\text {rev }}$ (Fig. 2b2). $E$ is higher in the region of the surrounding tissue below the tumor than the $E$ inside the tumor.

In the $Z X$ cross-section parallel to the electrodes, $E$ only exceeds the $E_{r e v}$ in the deepest part in of the tumor tissue inside model 1 (see $E$ distribution in direction $\mathrm{Z}$ in Fig. 2a3), while whole tumor volume inside model 2 is exposed to $E$ above $E_{\text {rev }}$ (Fig. 2b3).

We also calculated minimum and maximum electric field magnitudes Etumor ${ }_{\text {min }}$ and Etumor ${ }_{\text {max }}$ over the region representing the tumor, and the percentage of the tumor volume (Vtumor ${ }_{E \geq E \text { Erev }}$ ) exposed to the reversible electric field above $E>E_{\text {rev }}=400 \mathrm{~V} / \mathrm{cm}$, for both electrode placements analyzed (Table I).
Table I

Etumor $_{\text {max }}$ and Etumor min $_{\text {in }}$ inside the tumor and percentages of tumor volumes Vtumor $_{E \geq E \text { rev }}$ subjected to $E$ above $E_{\text {rev }}=$ $400 \mathrm{~V} / \mathrm{cm}$, calculated for both models.

\begin{tabular}{cccc}
\hline Model & $\begin{array}{c}\text { Etumor }_{\max } \\
{[\mathrm{V} / \mathrm{cm}]}\end{array}$ & $\begin{array}{c}\text { Etumor }_{\min } \\
{[\mathrm{V} / \mathrm{cm}]}\end{array}$ & Vtumor $_{E \geq \text { Erev }}$ \\
\hline 1 & 516 & 207 & $32 \%$ \\
2 & 1119 & 610 & $100 \%$ \\
\hline
\end{tabular}

In addition we calculated the percentage of healthy tissue volume exposed to $E$ above $E_{\text {rev }}\left(V_{s u r r} \operatorname{siErev}\right)$ and $E_{\text {irrev }}\left(\operatorname{Vsurr}_{E \geq E \text { Eirrev }}\right)$ over the entire volume of surrounding tissue in both models (Table II). Further, in order to more precisely investigate the local $E$ within the healthy surrounding tissue in the close vicinity of electrodes we calculated the average $E$ (Esurr) below the electrodes over the tissue exposed to E>Eirrev (Table II).

\section{Experimental Results}

The comparison between tumor regression obtained in the two groups of mice treated with ECT (Fig. 3) demonstrates

\section{Table II}

Average $E$ within surrounding tissue in close vicinity of electrodes (Esurr) and percentages of healthy tissue volumes $V_{s u r r}{ }_{E \geq E \text { Erev }}$ and Vsurr $_{E \geq \text { Eirrev }}$ subjected to $E$ above $E_{r e v}=400 \mathrm{~V} / \mathrm{cm}$ and Eirrev $=1500 \mathrm{~V} / \mathrm{cm}$, respectively.

\begin{tabular}{cccc}
\hline Model & $\begin{array}{c}\text { Esurr } \\
{[\mathrm{V} / \mathrm{cm}]}\end{array}$ & Vsurr $_{E \geq \text { Erev }}$ & Vsurr $_{\text {E Eirrev }}$ \\
\hline 1 & 2130 & $4.79 \%$ & $0.37 \%$ \\
2 & 1760 & $3.40 \%$ & $0.35 \%$ \\
\hline
\end{tabular}
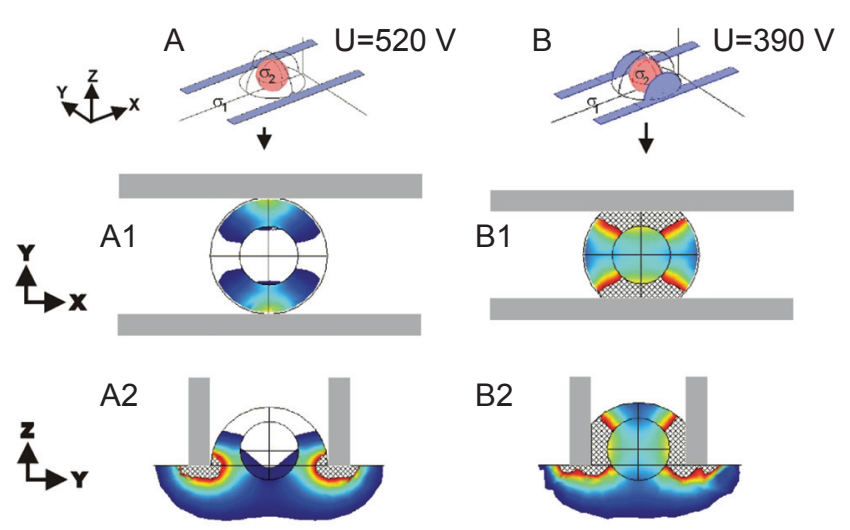

B2
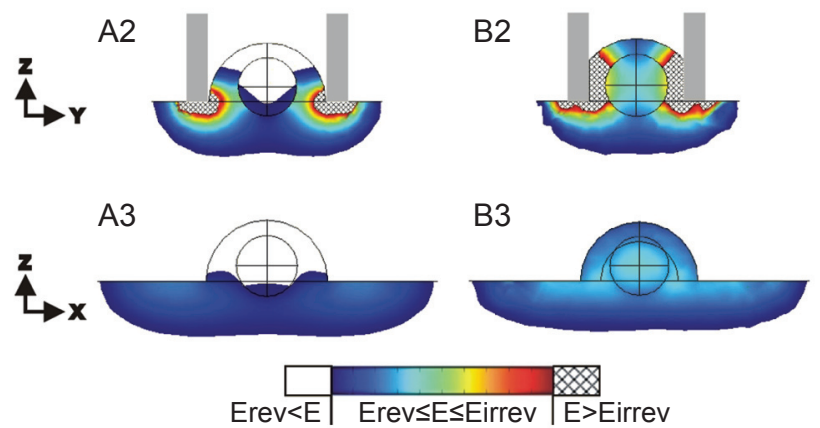

Figure 2: Comparison of results obtained between model 1 [(A) 3D model geometry with highlighted contact surface 1 ; (A1) $E$ in $X Y$ cross section; (A2) $E$ in $Z Y$ cross section; and (A3) $E$ in $Z X$ cross section] and model 2 [(B) $3 \mathrm{D}$ model geometry with highlighted contact surface 2; (B1) $E$ in $X Y$ cross section; (B2) $E$ in $Z Y$ cross section; and (B3) $E$ in $Z X$ cross section], $\left(\sigma_{1}=\right.$ $0.2 \mathrm{~S} / \mathrm{m}, \sigma_{2}=0.4 \mathrm{~S} / \mathrm{m}, E_{\text {rev }}=400 \mathrm{~V} / \mathrm{cm}$, and $\left.E_{\text {irrev }}=1500 \mathrm{~V} / \mathrm{cm}\right)$. 
that ECT performed with electrode placement with larger electrode-tissue contact surface (contact surface 2, $\mathrm{U}=390$ $\mathrm{V}, \mathrm{d}=3 \mathrm{~mm}$ ) resulted in significantly better anti-tumor effectiveness compared to the ECT with electrode placement with the smaller electrode tissue contact surface (contact surface $1, \mathrm{U}=520 \mathrm{~V}, \mathrm{~d}=4 \mathrm{~mm})(\mathrm{p}=0.0002)$. The average tumor volumes obtained at the end of the follow-up (on day 27) were $2697 \mathrm{~mm}^{3}$ and $648 \mathrm{~mm}^{3}$ for the groups of tumors treated with ECT with contact surfaces 1 and 2, respectively, while it was $4528 \mathrm{~mm}^{3}$ in the control group (statistically significant, $\mathrm{p}=0.006$ and $\mathrm{p}<0.0001$, respectively).

The ECT of tumors performed with electrode placement with contact surface 2 resulted in $7 \mathrm{CR}$ out of 15 treated mice (47\%) and in only $1 \mathrm{CR}$ in the group of 15 mice $(7 \%)$ treated with electrode placement with electrode contact tissue 1 (Table III). According to the Fisher's exact test, the difference between control group and the group treated with ECT (contact surface $2)$ is statistically significant $(p=0.007)$, while the difference between control group and the group of animals treated with ECT (contact surface 1 ) was not statistically significant $(\mathrm{p}=$ 1). Further, the result of Fisher's exact test evaluation between the group treated with ECT (contact surface 2) and the group treated with ECT (contact surface 1) is significant $(\mathrm{p}=0.04)$.

\section{Table III}

Number of CR in the three experimental groups. There were statistically significant differences between the ECT - contact surface 2 group and the two other groups $(p=0.007$ with the control group and $p=0.004$ with the ECT - contact surface 1 group). There was no significant difference between the control and the ECT - contact surface 1 groups.

\begin{tabular}{cccc}
\hline Groups & $\begin{array}{c}\text { Number } \\
\text { of mice }\end{array}$ & $\begin{array}{c}\text { Number } \\
\text { of CR }\end{array}$ & $\begin{array}{c}\text { Percentage } \\
\text { of CR }\end{array}$ \\
\hline Control & 13 & 0 & $0 \%$ \\
ECT - contact surface 1 & 15 & 1 & $7 \%$ \\
ECT - contact surface 2 & 15 & 7 & $47 \%$ \\
\hline
\end{tabular}

\section{Discussion}

In this study we numerically and experimentally analyzed the consequences of the effects of two different ways to place parallel plate electrodes for the treatment of cutaneous tumors by electrochemotherapy. These two situations differ by the contact surface between the electrodes and the tissue. The aim was to show which of the two electrode placements meets better the requirements for effective ECT, which means to achieve high electropermeabilization efficiency of target/ tumor tissue but at the same time spare normal tissue and avoid side effects. In terms of local electric field distribution the requirements are the following: (i) all the target tissue has to be exposed to the electric field $E$ above the threshold value for reversible electroporation $\left(E>E_{\text {rev }}\right)$ and (ii) the surrounding tissue should not be exposed to excessively high $E$, meaning that the local electric field in the surrounding healthy tissue should be as low as possible.
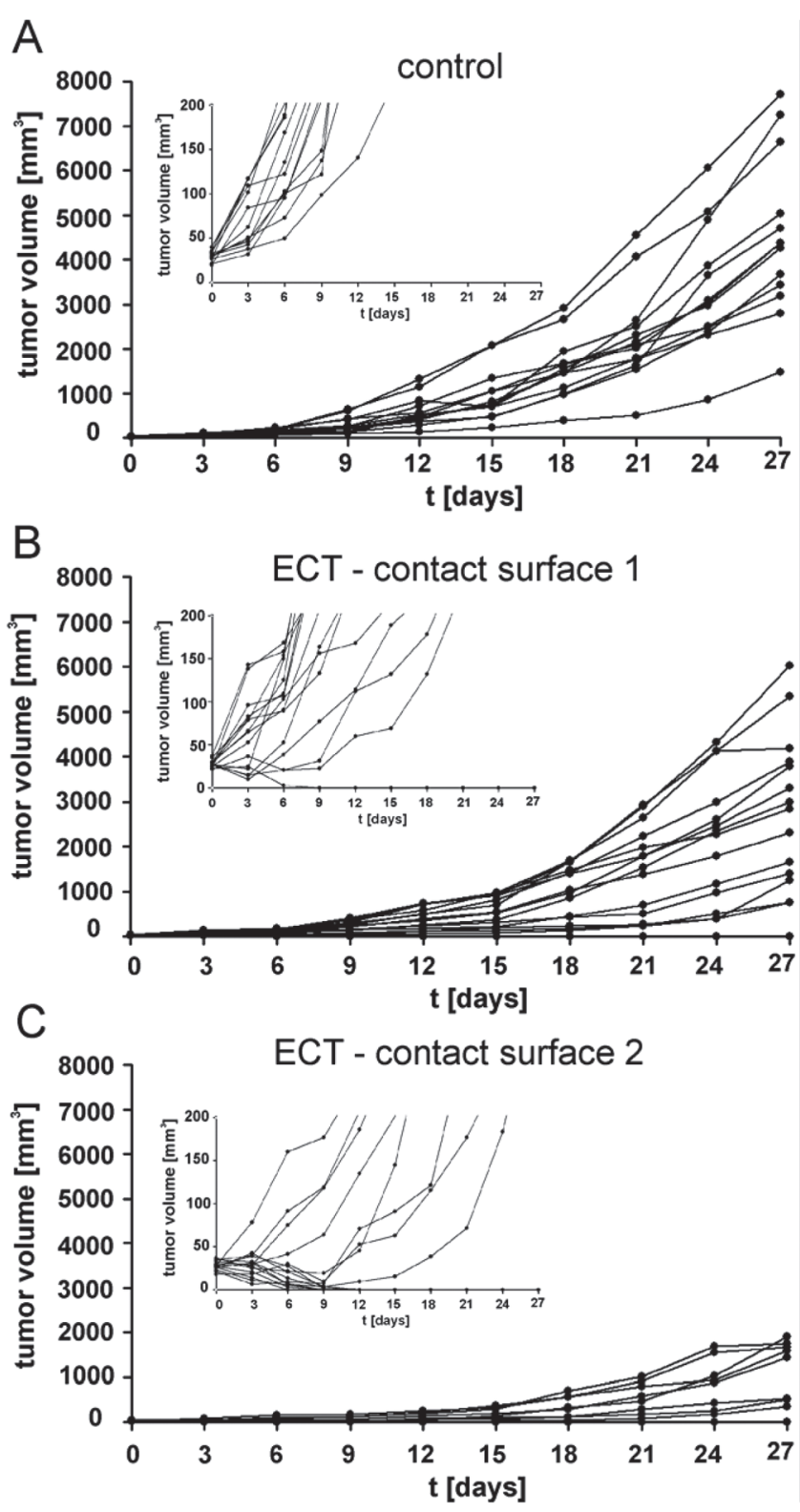

Figure 3: Evolution of the tumor volume: $(\mathbf{A})$ in the control group $(\mathrm{n}=$ 13); (B) in the mice treated with ECT $(\mathrm{U}=520 \mathrm{~V}, \mathrm{~d}=4 \mathrm{~mm}$, and contact surface 1) $(\mathrm{n}=15)$; and $(\mathbf{C})$ in the mice treated with ECT $(\mathrm{U}=390 \mathrm{~V}, \mathrm{~d}=$ $3 \mathrm{~mm}$, and contact surface 2$)(\mathrm{n}=15)$. Each curve corresponds to an individual tumor growth. Differences are statistically significant $(p=0.0002$ between the two treated groups and $p=0.006$ and $p<0.0001$ between the control group and, respectively, the groups treated with ECT using $d=4$ $\mathrm{mm}$ and contact surface 1 and $\mathrm{d}=3 \mathrm{~mm}$ and contact surface 2). Inserts in each panel show the growth of the tumors for sizes not larger than $200 \mathrm{~mm}^{3}$, displaying the initial tumor volume evolution and the regression of one and seven tumors, respectively, in the group treated with ECT using $\mathrm{U}=520 \mathrm{~V}$, $\mathrm{d}=4 \mathrm{~mm}$, and contact surface 1 and in the group treated with ECT using $\mathrm{U}$ $=390 \mathrm{~V}, \mathrm{~d}=3 \mathrm{~mm}$, and contact surface 2 .

The two 3D numerical models and the experiments with in vivo cutaneous tumors were carried out with the same input parameter as the voltage to distance ratio $U / d$ was set to $1300 \mathrm{~V} / \mathrm{cm}$, a parameter usually applied in clinical end ex- 
perimental ECT $(1,2,8)$. By means of numerical modeling we examined the influence of the dimensions of electrodetissue contact surface on the electric field strength inside the target tumor tissue (Tables I and II). The results show that the larger the electrode-tissue contact surface, the higher the local electric field strength inside the tumor and the lower local electric field in the surrounding tissue.

These numerical results were confirmed by in vivo ECT of mice tumors having similar dimensions as those numerically modeled. In the first electrode placement, the electrodes were $4 \mathrm{~mm}$ apart and just a point contact with the tumor convexity was obtained (corresponding to the point defined by the arrow in Fig. 1b). In the second electrode placement the electrodes were $3 \mathrm{~mm}$ apart. By "squeezing" the tissue in between the electrodes a larger electrode-tissue contact surface with cutaneous tumor convexity was obtained (corresponding to the patterned surface in Fig. 1d).

We obtained better results with the larger electrode-tissue contact surface 2, due to the fact that in this electrode placement the entire volume of the tumor was subjected to $E$ in the range $E_{\text {rev }} \leq E<E_{\text {irrev }}$. Indeed, the complete EPN of the tumor allowed the bleomycin to enter and kill all the tumor cells, resulting in tumor CR in 7 out of 15 treated tumors. In contrast, the smaller electrode-tissue contact surface resulted in only one CR out of 15 tumors, due to the residual untreated tumor cells, in the region of the tumor where the value of the local electric field strength was below $E_{\text {rev }}$.

It is interesting to note that the best antitumor (thus electropermeabilizing) effects in situation 2 are reached by the lowest voltage set $(U=390 \mathrm{~V}$ instead of $U=520 \mathrm{~V})$, just because in situation 2 the contact surface is the largest. This is in agreement with our previously published data where the local $E$ distribution in liver tissue was determined based on results of biological observations and numerical calculations obtained with needle electrodes of different diameters (14). The applied voltage at which reversible and irreversible tissue electropermeabilization was obtained was lower, and the local $E$ distribution was more homogeneous, using the electrodes of largest diameter, the needles of the largest diameter having of course the largest electrode tissue contact surface. Similarly, in this paper we show that in the larger electrode-tissue contact surface lower voltage $(U=390 \mathrm{~V})$ results in higher local $E$ inside the tumor (Table I), while lower value of $E$ in the close vicinity of the electrodes was found and less healthy tissue was electropermeabilized compared to the results obtained with the smaller contact surface (Tables I and II).

In conclusion, the experimental results presented herein are in good agreement with numerical models. By appropriate placement of electrodes on the surface of the treated tissue, a better coverage by sufficiently high electric field ( $E \geq$ Erev) can be achieved over the entire target tissue and minimized in the surrounding tissues and thus better therapy outcome. Recommendations can be given to researchers and physicians to choose electrodes geometry and placement in the way assuring the largest electrode-tissue contact surface. Another important consequence is that the needed parameters for successful ECT of each individual type of tumor can be determined, and thus the needed treatment planning carried out, by means of numerical modeling, as previously suggested in (17). Moreover, the electrode placement in a way to increase the electrode-tissue contact surface, described in this paper, can be beneficial also in other electropermeabilization mediated therapies such as gene electrotransfer (23), transdermal drug delivery (20), and irreversible tumor ablation (24-27).

\section{Acknowledgements}

This research was supported by the Slovenian Research Agency (ARRS), CNRS, IGR, French-Slovenian Scientific Cooperation Programme (PICS), and Science and Education Foundation of the Republic of Slovenia Ad-Futura.

\section{References}

1. Mir, L. M., Orlowski, S., Belehradek, J. Jr., Paoletti, C. Electrochemotherapy Potentiation of Antitumor Effect of Bleomycin by Local Electric Pulses. Eur J Cancer 27, 68-72 (1991).

2. Marty, M., Sersa, G., Garbay, J. R., Gehl, J., Collins, C. G., Snoj, M., Billard, V., Geertsen, P. F., Larkin, J. O., Miklavcic, D., Pavlovic, I., Paulin-Kosir, S. M., Cemazar, M., Morsli, N., Soden, D. M., Rudolf, Z., Robert, C., O'Sullivan, G. C., Mir, L. M. Electrochemotherapy - An Easy, Highly Effective and Safe Treatment of Cutaneous and Subcutaneous Metastases: Results of ESOPE (European Standard Operating Procedures of Electrochemotherapy) Study. Eur J Cancer Supplements 4, 3-13 (2006).

3. Snoj, M., Rudolf, Z., Paulin-Kosir, S. M., Cemazar, M., Snoj, R., Sersa, G. Long Lasting Complete Response in Melanoma Treated by Electrochemotherapy. Eur J Cancer Supplements 4, 26-28 (2006).

4. Garbay, J. R., Billard, V., Bernat, C., Mir, L. M, Morsli, N., Robert, C. Successful Repetitive Treatments by Electrochemotherapy of Multiple Unresectable Kaposi Sarcoma Nodules. Eur J Cancer Supplements 4, 29-31 (2006).

5. Whelan, M. C., Larkin, J. O., Collins, C. G., Cashman, J., Breathnach, O., Soden, D. M., O'Sullivan, G. C. Effective Treatment of an Extensive Recurrent Breast Cancer Which Was Refractory to Multimodal Therapy by Multiple Applications of Electrochemotherapy. Eur J Cancer Supplements 4, 32-34 (2006).

6. Gehl, J., Geertsen, P. F. Palliation of Heamorraging and Ulcerated Cutaneous Tumors Using Electrochemotherapy. Eur J Cancer Supplements 4, 35-37 (2006).

7. Sersa, G. The State-of-the-art of Electrochemotherapy Before the ESOPE Study; Advantages and Clinical Uses. Eur J Cancer Supplements 4, 52-9 (2006).

8. Mir, L. M, Gehl, J., Sersa, G., Collins, C. G., Garbay, J. R., Billard V., Geertsen, P. F., Rudolf, Z., O’Sullivan, G. C., Marty, M. Standard Operating Procedures of Electrochemotherapy: Instructions for the Use of Bleomycin or Cisplatin Administered either Systemically or Locally and Electric Pulses Delivered by CliniporatorTM by Means of Invasive or Non-invasive Electrodes. Eur J Cancer Supplements 4, 14-25 (2006). 
9. Mir, L. M., Orlowski, S. Mechanisms of Electrochemotherapy. Adv Drug Deliv Rev 35, 107-118 (1999).

10. Miklavcic, D., Corovic, S., Pucihar, G., Pavselj, N. Importance of Tumor Coverage by Sufficiently High Local Electric Field for Effective Electrochemotherapy. Eur J Cancer Supplements 4, 45-51 (2006).

11. Mir, L. M. Bases and Rationale of Electrochemotherapy. Eur J Cancer Supplements 4, 38-44 (2006).

12. Gehl, J., Sorensen, T. H., Nielsen, K., Raskmark, P., Nielsen, S. L., Skovsgaard, T., Mir, L. M. In vivo Electroporation of Skeletal Muscle: Threshold, Efficacy and Relation to Electric Field Distribution. Biochim Biophys Acta 1428, 233-240 (1999).

13. Corovic, S., Pavlin, M., Miklavcic, D. Analytical and Numerical Quantification and Comparison of the Local Electric Field in the Tissue for Different Electrode Configurations. BioMedical Engineering OnLine 6, 1-14 (2007).

14. Miklavcic, D., Semrov, D., Mekid, H., Mir, L. M. A Validated Model of In vivo Electric Field Distribution in Tissues for Electrochemotherapy and for DNA Electrotransfer for Gene therapy. Biochim Biophys Acta 1523, 73-83 (2000).

15. Pavlin, M., Pavselj, N., Miklavcic, D. Dependence of Induced Transmembrane Potential on Cell density, Arrangement, and Cell Position Inside a Cell System. IEEE Trans Biomed Eng 49, 605-612 (2002).

16. Pavselj, N., Bregar, Z., Cukjati, D., Batiuskaite, D., Mir, L. M., Miklavcic, D. The Course of Tissue Permeabilization Studied on a Mathematical Model of a Subcutaneous Tumor in Small animals. IEEE Trans Biomed Eng 52, 1373-1381 (2005).

17. Sel, D., Lebar, A. M., Miklavcic, D. Feasibility of Employing ModelBased Optimization of Pulse Amplitude and Electrode Distance for Effective Tumor Electropermeabilization. IEEE Trans Biomed Eng 54, 773-781 (2007).
18. Ivorra, A., Rubinsky, B. In vivo Electrical Impedance Measurements During and After Electroporation of Rat Liver. Bioelectrochemistry 70, 287-295 (2007).

19. Miklavcic, D., Pavselj, N., Hart, X. F. Electric Properties of Tissues. Wiley Encyclopedia of Biomedical Engineering, John Wiley \& Sons, New York (2006).

20. Pliquett, U. F., Vanbever, R., Preat, V., Weaver, J. C. Local Transport Regions (LTRs) in Human Stratum Corneum Due to Long and Short 'high voltage' Pulses. Bioelectrochem Bioenerg 47, 151-161 (1998).

21. (UKCCCR) Guidelines for Welfare of Animals in Experimental Neoplasia (Second Edition). Br J Cancer 77, 1-10 (1998).

22. Zupanic, A., Ribaric, S., Miklavcic, D. Increasing the Repetition Frequency of Electric Pulse Delivery Reduces Unpleasant Sensations that Occur in Electrochemotherapy. Neoplasma 54, 246-250 (2007).

23. Cukjati, D., Batiuskaite, D., Andre, F., Miklavcic, D., Mir, L. M. Real Time Electroporation Control for Accurate and Safe In vivo Nonviral Gene Therapy. Bioelectrochemistry 70, 501-507 (2007).

24. Al-Sakere, B., Bernat, C., Connault, E., Opolon, P., Rubinsky, B. Davalos, R., Mir, L. M. Tumor Ablation with Irreversible Electroporation. PLOS ONE 2, 11 (2007).

25. Al-Sakere, B., Bernat C., André, F., Connault, E., Opolon, P., Davalos, R. V., Mir, L. M. A Study of the Immunological Response to Tumor Ablation with Irreversible Electroporation. Technol Cancer Res Treat 6, 301-306 (2007).

26. Onik, G., Mikus, P., Rubinsky, B. Irreversible Electroporation: Implications for Prostate Ablation. Technol Cancer Res Treat 6, 295 300 (2007)

27. Bertacchini, C., Margotti, P. M., Bergamini, E., Lodi, A., Ronchetti, M., Cadossi, R. Design of an Irreversible Electroporation System for Clinical Use. Technol Cancer Res Treat 6, 313-320 (2007).

Received: April 1, 2008; Revised: June 7, 2008, Accepted: July 25, 2008 